\title{
Negative Thermal Expansion of Diamond and Zinc-Blende Semiconductors
}

\author{
Slawomir Biernacki ${ }^{(a)}$ and Matthias Scheffler \\ Fritz-Haber-Institut der Max-Planck-Gesellschaft, Faradayweg 4-6, \\ D-1000 Berlin 33, Federal Republic of Germany \\ (Received 13 March 1989)
}

\begin{abstract}
Experimentally it is well known that diamond and zinc-blend semiconductors show an "unusual" (i.e., negative) thermal expansion at about $100 \mathrm{~K}$. We performed density-functional-theory calculations of thermodynamic potentials (i.e., total energies and entropies) for perfect crystals, to study the temperature dependence of the lattice parameter. The origin of the negative expansion effect is traced back to the entropy contribution of the Gibbs free energy.
\end{abstract}

PACS numbers: $64 \cdot 10 .+\mathrm{h}, 65.50 .+\mathrm{m}, 65.70 .+\mathrm{y}$

For diamond and zinc-blende semiconductors the linear thermal expansion coefficient, $\alpha=(1 / a)(\partial a / \partial T)_{p}$, where $a$ is the crystal lattice parameter, is not a constant of temperature, and at low temperatures it even becomes negative. ${ }^{1}$ Thus, the lattice contracts upon heating. Figure 1 shows the experimental result for silicon as open circles. ${ }^{2}$ In this paper we present a parameter-free approach to calculate thermodynamic potentials from first principles and we use the method to study the temperature dependence of the crystal lattice parameter, taking silicon as an example. The calculations reproduce the effect of negative thermal expansion and they allow a microscopic analysis of its origin.

For a given temperature and pressure the equilibrium state of a perfect or imperfect crystal is determined by the Gibbs free energy,

$$
G\left(N_{A}, N_{B}, \ldots, T, p\right)=U-T S+p V .
$$

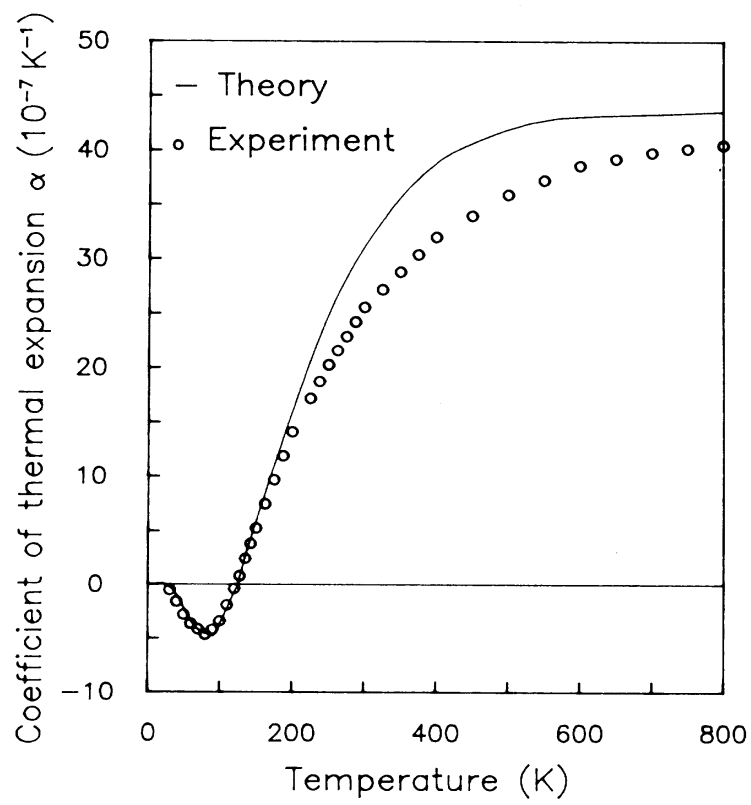

FIG. 1. Coefficient of thermal expansion for crystalline silicon as a function of temperature. Experimental data (open circles) are from Ref. 2.
Here $N_{A}, N_{B}, \ldots, T, p, U, S$, and $V$ are the numbers of particles (atoms and electrons) of type $A, B, \ldots$, temperature, pressure internal energy, entropy, and crystal volume. Particle numbers, temperature, and pressure are determined by the experimental situation. The detailed atomic structure (in thermodynamic equilibrium) and its internal energy, entropy, and volume follow from the condition that $G$ must be at a minimum of all possible atomic arrangements and electronic states.

In our calculations of the Gibbs free energy we take the static and vibrational contributions into account. Electronic excitations will be neglected because they contribute only at higher temperatures. Equation (1) can be evaluated if the electron ground-state total energy, $U^{\text {static }}$, and all vibrational frequencies, $\omega_{n}(\mathbf{k})$, are known (see Refs. 3 and 4). From $d G / d a=0$ it follows that ${ }^{1,5}$

$$
\alpha=\frac{1}{3 B_{0}} \sum \gamma_{n}(\mathbf{k}) c_{v, n}(\mathbf{k}) .
$$

The sum goes over all vibrational modes. The $\gamma_{n}(\mathbf{k})$ are the mode Grüneisen parameters $\left\{\gamma_{n}(\mathbf{k})=-d\left[\ln \omega_{n}(\mathbf{k})\right] /\right.$ $d[\ln V]\}$ and the $c_{v}, n(\mathbf{k})$ are the contributions of the modes $|n, \mathbf{k}\rangle$ to the specific heat

$$
c_{r, n}(\mathbf{k})=\left[\hbar \omega_{n}(\mathbf{k}) / V\right] d\left\{\exp \left[\hbar \omega_{n}(\mathbf{k}) / k_{B} T\right]-1\right\}^{-1} / d T .
$$

Equation (2) shows that a negative thermal expansion can arise if some modes have a negative $\gamma_{n}(\mathbf{k}){ }^{1,6-8}$ Previous (empirical) calculations have evaluated Eq. (2), taking only the explicit $T$ dependence of the $c_{r, n}(\mathbf{k})$ into account. We take a different approach: Instead of evaluating the derivatives of the individual $\omega_{n}(\mathbf{k})$, we calculate the Gibbs free energy as a function of lattice parameter and then determine its minimum (see Ref. 4). The calculations are performed using density-functional theory (DFT) in the local-density approximation for exchange and correlation and first-principles pseudopotentials. ${ }^{9}$ The phonon energies $\hbar \omega_{n}(\mathbf{k})$ are evaluated by diagonalizing the dynamical matrix $D_{i j}=d^{2} U^{\text {static }} / d \mathbf{R}_{i}$ $\times d \mathbf{R}_{j}$. As the $D_{i, j}$ change with lattice parameter, anharmonic effects are included in the calculations. ${ }^{10}$ As a consequence, the vibrational frequencies and also the mode Grüneisen parameters $\gamma_{n}(\mathbf{k})$, the bulk modulus $B_{0}$, 
and the $c_{v, n}(\mathbf{k})$ become volume dependent and by this also (indirectly) temperature dependent. This has usually been neglected in calculations based on approximate methods, ${ }^{1,7,8}$ but it becomes important for temperatures above $300 \mathrm{~K}$.

The dynamical matrix can be calculated directly from many DFT calculations or it can be calculated from an expansion of the DFT total energy $U^{\text {static }}\left(\left\{\mathbf{R}_{i}\right\}\right)$ for any lattice parameter in an analytic form, which we need to know only up to second order in the atomic displacements. We take the second approach. As described in Ref. $4, D_{i, j}$ can be obtained from the bulk modulus $B_{0}$ and the breathing-mode force-constant $k_{b}$. The latter corresponds to an artificial distortion of the $\mathrm{Si}$ crystal lattice, where the four neighbors of an atom are displaced in a breathing-mode fashion. All other atoms are kept fixed at their perfect-crystal positions. Note that both quantities are second derivatives of the total energy, as is the dynamical matrix. Both quantities probe different aspects of the crystal: $B_{0}$ corresponds to a longwavelength distortion $[\mathbf{k}=(0,0,0)]$ and $k_{b}$ corresponds to a wave packet of short-wavelength distortions.

Although the phonon dispersion curves obtained by the method of Ref. 4 have some well-known deficiencies (see the flattening of the experimental bands at $X$ and $L$ in Fig. 4), it turns out that the derivative of the Gibbs free energy with respect to the crystal lattice parameter is quite accurate. $B_{0}$ is known from theory and experiment but $k_{b}$ can be obtained from theory only. Our DFT calculations [plane-wave basis $\left(E_{\text {cut }}=20 \mathrm{Ry}\right)$, twoatom cell, ten special $\mathbf{k}$ points] give the following results at $T=0 \mathrm{~K}: a_{0}=5.385 \AA$ (experiment, $5.43 \AA$ ), $B_{0}$ $=0.947 \mathrm{Mbar}$ (experiment, $0.992 \mathrm{Mbar}$ ), and $\boldsymbol{B}_{0}^{\prime}=3.93$ (experiment, 4.2). The effect of zero-point vibrations is included in these values. Figure 2 shows the $T=0 \mathrm{~K}$ equation of state [Eq. (1)]. It also shows how $B_{0}$ and $k_{b}$ depend on the lattice parameter. These curves are slightly nonlinear functions of the lattice parameter. The numerical accuracy of this nonlinearity is not very high, which affects the accuracy of our results at higher temperature $(T \geq 300 \mathrm{~K})$. From $D_{i, j}$ we obtain the vibrational modes (again as functions of the lattice parameter). We then evaluate the Gibbs free energy at normal pressure, and from its minimum as a function of lattice parameter the theoretical curve in Fig. 1 is obtained.

The negative expansion is indeed reproduced by the theory (see Fig. 1). The origin of this effect lies in the temperature-dependent contributions of the Gibbs free energy which are displayed in Fig. 3 for three characteristic temperatures. The Gibbs free energy is the sum of the $T=0 \mathrm{~K}$ equation of state (left-hand side of Fig. 2) and the bottom row of Fig. 3. It is worth noting that the entropy term dominates the temperature-dependent contributions of the Gibbs free energy at all temperatures. Thus, the thermal expansion, including the range where it is negative, is an entropy-driven effect. The slope of the vibrational entropy $-S^{\mathrm{vib}} T$ (and of $U^{\mathrm{vib} *}-S^{\mathrm{vib}} T$ ) is found to be negative at $T=10 \mathrm{~K}$, positive at $T=80 \mathrm{~K}$, and negative at $T=500 \mathrm{~K}$. Therefore, the minimum of the $T=0 \mathrm{~K}$ Gibbs free energy (i.e., of $U^{\text {static }}+U^{\text {vib0 }}$ in Fig. 2) is shifted to a larger value of lattice parameter at $T=10 \mathrm{~K}$, to a smaller value at $T=80 \mathrm{~K}$, and to a larger value at $T=500 \mathrm{~K}$. The shift at $T=10 \mathrm{~K}$ is very small and cannot be resolved in Fig. 1 .

Figure 4 gives a schematic summary of why the slope of $-S^{\mathrm{vib}} T$ changes sign for different temperatures:

At high temperatures, both the acoustic and the optical branches of the phonon band structure are occupied. We may therefore replace the whole band structure by one average energy $\hbar \bar{\omega}$. Because the interatomic coup-
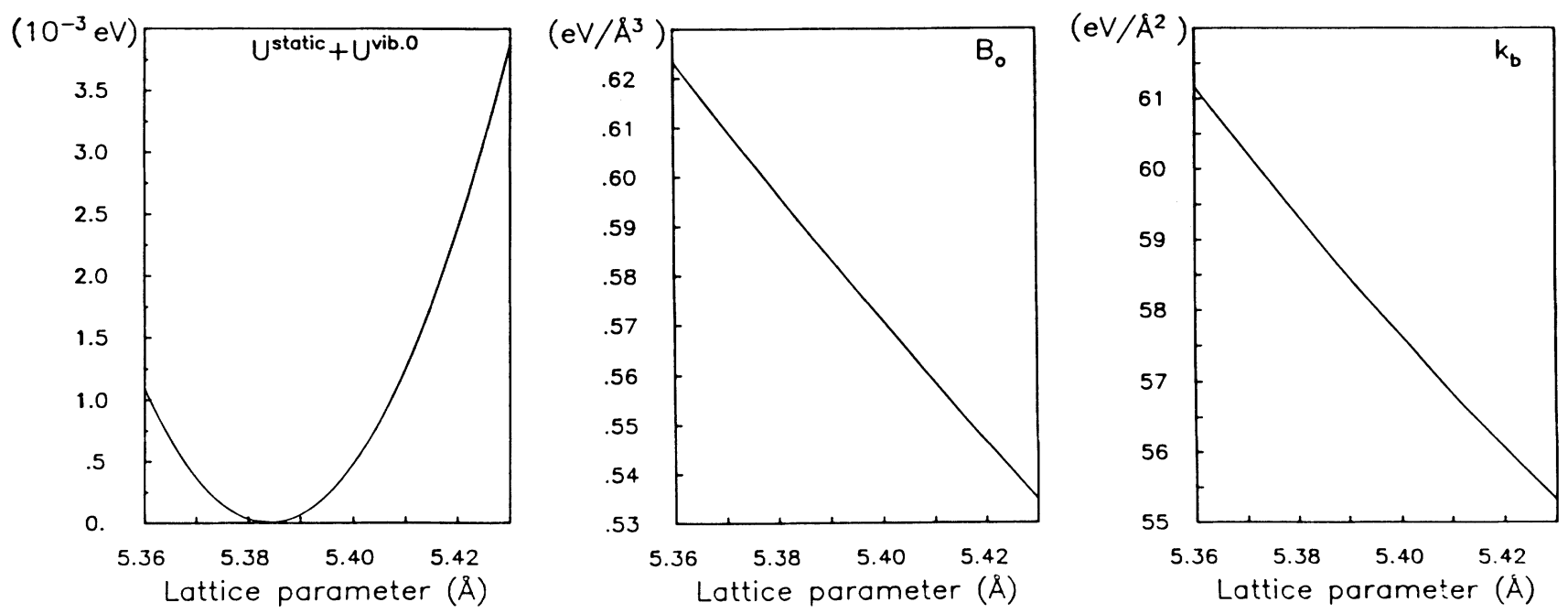

FIG. 2. Equation of state at $T=0 \mathrm{~K}$ (i.e., $U^{\text {static }}+U^{\text {vib } 0}$ ), the bulk modulus $B_{0}$, and the breathing-mode force constant $k_{b}$ for a $\mathrm{Si}$ crystal as a function of lattice parameter, as derived from density-functional-theory calculations. 

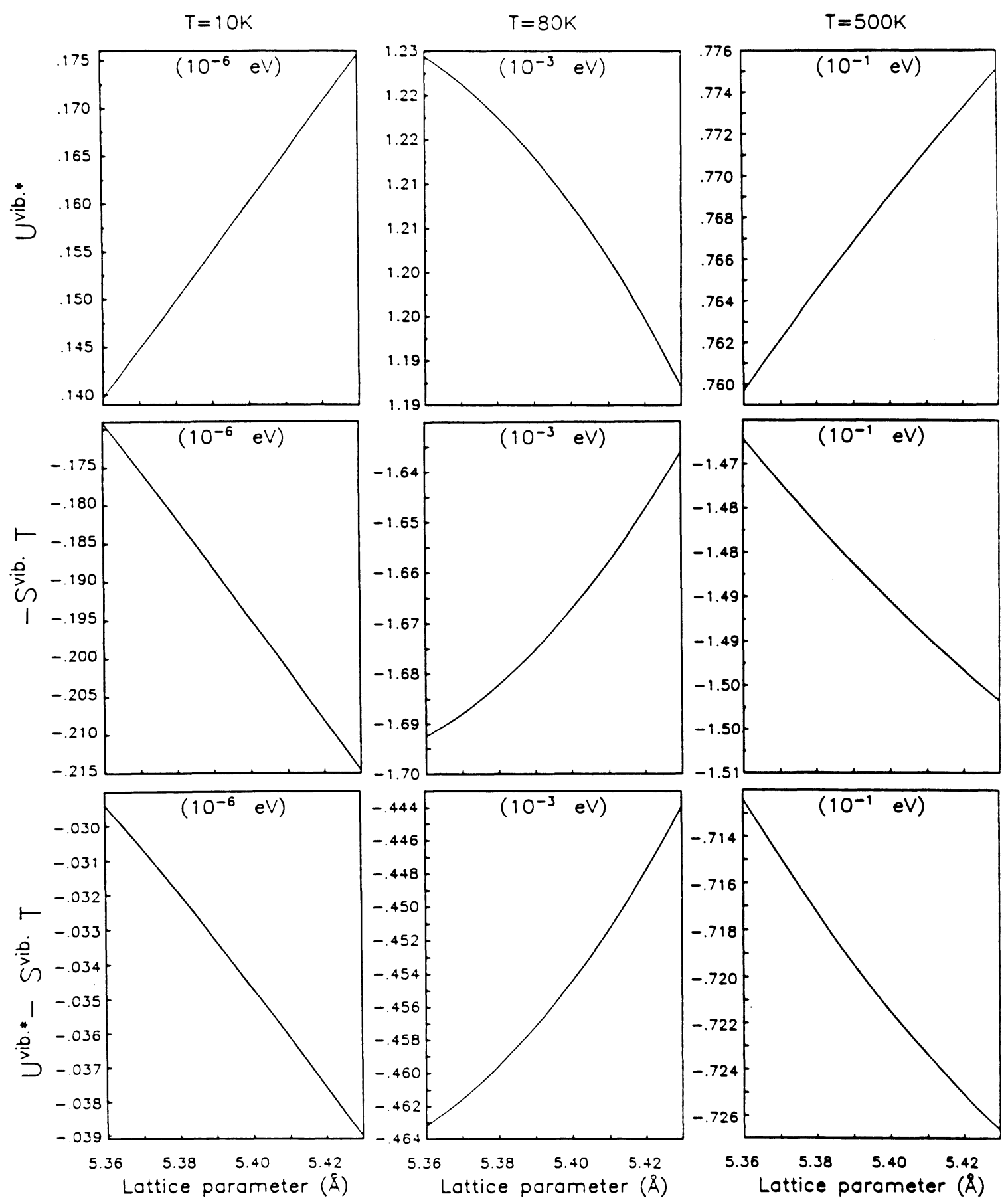

FIG. 3. The temperature-dependent contributions to the Gibbs free energy at three characteristic temperatures for crystalline silicon. The bottom row shows the vibrational Helmholtz free energy without zero-point vibrations.

ling decreases with increasing interatomic distances, this average frequency decreases with increasing lattice parameter (see right-hand side of Fig. 4). Thus, with increasing lattice parameter more phonons get thermally occupied and the entropy increases; i.e., the slope of $-S^{\mathrm{vib}} T$ is negative and the lattice expands.

At $T=80 \mathrm{~K}$, only phonons with an energy in the lower half of the band structure are excited. This implies that the high density of states of the transverse acoustic (TA) branch at $X$ and $L$ is important. With increasing lattice parameter, the hybridization between the acoustic and optical branches becomes smaller, which implies that the hybridization 


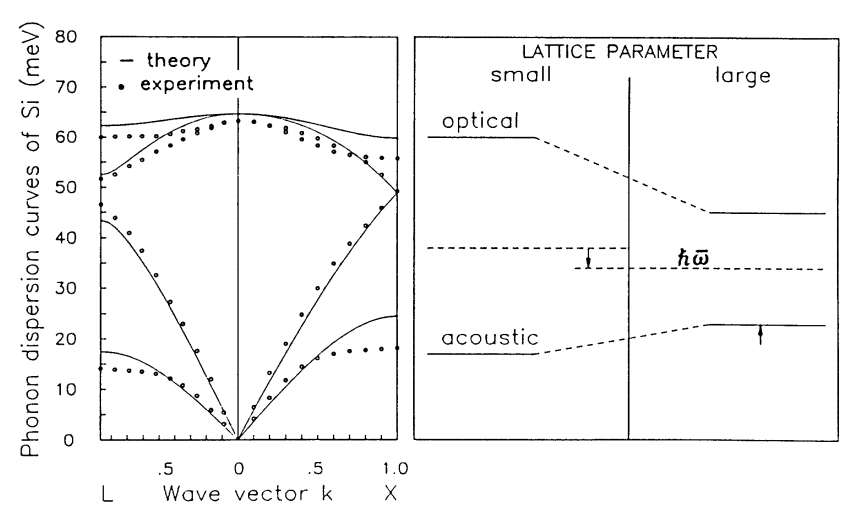

FIG. 4. Schematic explanation of the thermal expansion of silicon. The left-hand side shows the phonon band structure as it follows from our parameter-free approach (see text) in comparison with experimental results (Ref. 11). The right-hand side shows the average phonon energy, $\hbar \bar{\omega}$, which is relevant at high temperatures, at which the whole phonon band structure is thermally occupied. Furthermore, it shows the hybridization band gap between the acoustic and optical band. This gap decreases with increasing lattice parameter. Then the acoustic "level" shifts to higher energies with increasing lattice parameter.

gap decreases and the TA $(X)$ and TA $(L)$ energies move to higher energy (see Fig. 4). In other words, the Grüneisen parameters of the zone-boundary phonons TA $(X)$ and TA $(L)$ are negative. As the density of states is shifted to higher energies, fewer phonons get excited. The entropy therefore decreases with increasing lattice parameter and $-S^{\text {vib }} T$ has a positive slope. Therefore, the lattice contracts compared to the $T=0 \mathrm{~K}$ lattice parameter.

At very low temperatures, only the acoustic branch at $\Gamma$ is occupied. Here the bands get flatter with increasing lattice parameter. As a consequence, more phonons get occupied and thus the entropy increases with increasing lattice parameter. Thus, the slope of $-S^{\mathrm{vib}} T$ is negative and the lattice expands.

Our calculations reproduce the experimentally known effect of negative thermal expansion (see Fig. 1) and they provide a detailed microscopic explanation. Quantitatively the agreement between theory and experiment is not too bad: Note that we are dealing with very small changes of the lattice parameter (of order $10^{-4} \AA$ ) and that our theoretical bulk modulus, as determined by a converged DFT calculation, is about $4.5 \%$ smaller than the experimental value. Therefore, one can hardly expect better quantitative agreement between a parameter-free theory and experiment. The same qualitative behavior of the thermal expansion should occur for all systems with two atoms per unit cell, provided that the acoustical- and optical-phonon branches are well separated.

We gratefully acknowledge discussions with U. Scherz, J. Furthmüller, and R. Gillert. Part of this work was supported by the Deutsche Forschungsgemeinschaft.

\footnotetext{
(a) Permanent address: Institute of Physics (PAN), Al. Lotnikow 46, 02-668 Warsaw, Poland.
}

${ }^{1}$ G. Dolling and R. A. Cowley, Proc. Phys. Soc. 88, 463 (1966).

${ }^{2}$ H. Ibach, Phys. Status Solidi 31, 625 (1969).

${ }^{3} \mathrm{M}$. Born, Atomtheorie des festen Zustandes (Teubner, Leipzig, Berlin, 1923), 2nd ed., Eqs. (288) and (289).

${ }^{4}$ M. Scheffler and J. Dabrowski, Philos. Mag. A 58, 107 (1988).

${ }^{5}$ N. W. Ashcroft and N. Mermin, Solid State Physics (Holt-Saunders Japan, Tokyo, 1981), Eq. (25.20).

${ }^{6}$ M. Blackman, Proc. Phys. Soc. B 70, 827 (1957).

${ }^{7}$ H.-Matsuo Kagaya, Y. Kitani, and T. Soma, Solid State Commun. 58, 399 (1986).

${ }^{8}$ M. Delannoy-Coutris and G. Perrin, Phys. Status Solidi (b) 138, 93 (1986).

${ }^{9}$ G. B. Bachelet, D. R. Hamann, and M. Schlüter, Phys. Rev. B 26, 4199 (1982).

${ }^{10}$ For compound semiconductors it is important to include also an ionic contribution in $D_{i, j}$ (due to effective charges). See, for example, M. Scheffler and U. Scherz, in Defects in Semiconductors, edited by H. J. von Bardeleben, Materials Science Forum Series Vols. 10-12 (Trans Tech, Switzerland, 1986), p. 353.

${ }^{11}$ Physics of Group IV and III-V Compounds, edited by $O$. Madelung, Landolt-Börnstein: Numerical Data and Functional Relationships in Science and Technology (Springer-Verlag, Berlin, 1982), Group 3, Vol. 17, Part a. 